\title{
MODELAGEM NUMÉRICA DO IMPACTO AMBIENTAL ASSOCIADO À APLICAÇÃO DE FOSFOGESSO COMO COBERTURA DE ATERROS SANITÁRIOS
}

\author{
NUMERICAL MODELING OF THE ENVIRONMENTAL IMPACT \\ ASSOCIATED TO THE APPLICATION OF PHOSPHOGYPSUM AS \\ LANDFILL COVER
} Stela Dalva Santos Cota ${ }^{1}$, Vanusa Maria Feliciano Jacomino ${ }^{1}$, Maria Helena Tirollo Taddei ${ }^{2}$,
Marcos Roberto Nascimento

Artigo recebido em: 12/11/2011 e aceito para publicação em: 07/05//2012

\begin{abstract}
Dihydrate calcium sulphate (phosphogypsum) is a residue of phosphoric acid production that has been used in Brazil for agricultural purposes. However, the rate of generation of phosphogypsum is much higher than the rate of its use in agriculture and, therefore, much of this material has been stored in piles near where it is produced. This paper investigates whether the application of phosphogypsum as a material for landfill cover can contribute to an increase in exposure of humans to the natural radioactivity and toxic metals, through experimental studies (anaerobic biodegradation of the solid waste/phosphogypsum mixture) and mathematical modeling of contaminant transport in aquifers. The results indicated that application of phosphogypsum in landfill cover does not result in significant risks to either human health or the environment, for the examined conditions.
\end{abstract}

Keywords: Numerical modeling. Landfill. Phosphogypsum. Environmental contamination. Aquifer.

Resumo: Como resíduo do processo de produção de ácido fosfórico tem-se o sulfato de cálcio dihidratado (fosfogesso), que, no Brasil, vem sendo usado para fins agrícolas. Entretanto, a taxa de geração do fosfogesso ainda é muito maior que a taxa de utilização na agricultura e, portanto, grande parte do material vem sendo armazenada em pilhas no próprio local de produção. O presente trabalho teve por objetivo investigar, por meio de estudos experimentais (biodegradação anaeróbica da mistura lixo urbano e fosfogesso) e da modelagem matemática de transporte de contaminantes no aquífero, se o uso do fosfogesso como material de cobertura em aterros sanitários contribui para um aumento da exposição de seres humanos à radioatividade natural e metais. Os resultados encontrados indicaram que, para as condições analisadas, a aplicação do fosfogesso como material de cobertura de aterros sanitários não resulta em riscos significativos à saúde humana ou ao meio ambiente.

Palavras Chave: Modelagem numérica. Aterros sanitários. Fosfogesso. Contaminação ambiental. Aquífero.

\section{INTRODUÇÃO}

O fosfogesso, subproduto da indústria de fertilizantes fosfatados, é produzido em grande escala no Brasil. Estima-se que até meados de 1991, a quantidade de fosfogesso estocada no Brasil era de cerca de 30 milhões de toneladas. Embora a composição do fosfogesso seja basicamente de cálcio dihidratado, o material pode conter níveis elevados de impurezas que provêm da rocha fosfática que é usada como matéria prima na produção de fertilizantes. Entre as impurezas, têm-se os metais (por exemplo, $\mathrm{Cd}, \mathrm{As}, \mathrm{Pb}, \mathrm{Cd}$ e $\mathrm{Zi}$ ), os fluoretos e os radionuclídeos das séries naturais do urânio e tório (SANTOS, 2002).
A forma mais comum de descarte deste resíduo é a sua disposição em pilhas em áreas próximas às fábricas. Entretanto, esta prática pode representar um risco potencial de contaminação ambiental, principalmente, para o ar, solo e para as fontes de água localizadas nas proximidades das mesmas. No Brasil, este material vem sendo utilizado há várias décadas, em especial, como insumo agrícola. Nesse caso, o fosfogesso ou o "gesso agrícola" é largamente aplicado como fonte de cálcio e enxofre, como condicionador de subsuperfície e para correção de solos saturados com sódio ou potássio (OLIVEIRA, 2008).

\footnotetext{
${ }^{1}$ Centro de Desenvolvimento da Tecnologia Nuclear - CDTN/CNEN, Campus UFMG, Belo Horizonte, MG (sdsc@cdtn.br, vmfj@cdtn.br)

${ }^{2}$ Laboratório de Poços de Caldas - LAPOC/CNEN, Poços de Caldas, MG (mhtaddei@cnen.gov.br, pmarcos@cnen.gov.br)
} 
Entretanto, a taxa de geração do fosfogesso ainda é muito maior que a taxa de utilização na agricultura e, portanto, grande parte desse material vem sendo armazenada em pilhas, no próprio local de produção.

Uma das alternativas que poderia contribuir para o equilíbrio entre a geração e a aplicação do fosfogesso é a sua utilização em aterros sanitários, que constituem uma forma para a deposição final de resíduos sólidos gerados pela atividade humana. Neles são dispostos resíduos domésticos, comerciais, de serviços de saúde, da indústria de construção, ou dejetos sólidos retirados do esgoto.

Existem poucos trabalhos associados ao uso do fosfogesso como material de cobertura de aterros sanitários, sendo que os estudos disponíveis estão voltados, predominantemente, para a aplicação desse resíduo na agricultura e na construção civil (CANUT, 2006; PAPASTEFANOU et al., 2006; PÉREZ-LÓPEZ et al., 2007, MÁDUAR et al, 2011; GODINHO-CASTRO et al., 2012). Entretanto, estudos realizados no Instituto de Pesquisas do Fosfato da Florida (SHIEH, 2011) demonstraram que o fosfogesso, por ser um composto enriquecido com altos teores de sulfato de cálcio $\left(\mathrm{CaSO}_{4}\right)$, pode ser utilizado em um ambiente anaeróbico, como os encontrados nos aterros sanitários, para acelerar processos microbiológicos de decomposição dos resíduos sólidos municipais e, portanto, aumentar a vida útil dessas instalações.

Quando é feita a deposição de resíduos sólidos em um aterro, processos biológicos, químicos e físicos modificam os resíduos. Nesse processo, entretanto, algumas das substâncias tóxicas depositadas não são de rápida degradação (por exemplo, metais pesados e radioativos de meia vida longa). Assim, nos primeiros anos após a deposição desses contaminantes, muito pouco do conteúdo original é lixiviado, o que torna os depósitos uma fonte poluidora potencial. Como os componentes radioativos são passíveis de liberação por séculos após sua deposição, a preocupação é que a mobilidade de metais e elementos radioativos de meia vida longa possa aumentar conforme se modifique as condições do aterro e resulte em problemas ambientais e riscos à saúde da população (BIRDSELL et al, 2000).
Conseqüentemente, para qualquer prática de deposição é crucial demonstrar-se que a escolha do sistema para dispor os resíduos é adequada. Isto é geralmente alcançado a partir da demonstração do atendimento dos critérios de segurança estabelecidos em normas. Nesse caso, a avaliação de segurança é um componente chave para verificar a aceitabilidade de uma determinada prática de deposição. O objetivo de uma avaliação de segurança, após a deposição final de um resíduo contendo metais e radionuclídeos das séries do urânio e tório em um aterro sanitário, é determinar o impacto que ocasionará no ser humano e no meio ambiente como uma função do tempo (PONTEDEIRO, 2006).

No caso de deposição de resíduos, essa análise é realizada por meio da utilização de modelos numéricos para prognosticar como as concentrações dos poluentes podem variar nas águas subterrâneas ao longo do tempo e da distância, devido à atenuação e degradação dos contaminantes. Existem diversos modelos que realizam esse tipo de simulação, tais como o programa MODFLOW (HARBAUGH, 2005), desenvolvido pela U.S. Geological Survey para a simulação do fluxo em meios saturados, e o programa em elementos finitos HYDRUS (VOGEL et al., 1996) para a simulação de fluxo de água em zona não saturada.

Dentro deste contexto, a maior contribuição do presente trabalho consta da realização de um estudo de avaliação de segurança para obtenção de informações sobre o comportamento de contaminantes radioativos naturais e metais no aquífero em decorrência da aplicação do fosfogesso como cobertura de aterros sanitários. Para tanto, foi implementada uma metodologia para estimativa de aportes de contaminantes no sistema hídrico natural devido à percolação através da camada de argila de um aterro sanitário e seu transporte através de um aqüífero superficial até sua descarga em um curso d'água superficial. $O$ trabalho envolveu também a caracterização do resíduo fosfogesso e a determinação, em condições de laboratório, da proporção ideal de mistura resíduo sólido urbano/fosfogesso a ser aplicada no aterro sanitário visando o aumento da sua vida útil. 


\section{MATERIAIS E MÉTODOS}

\section{Caracterização do resíduo fosfogesso}

Amostras de fosfogesso foram coletadas na Unidade da Fosfértil/Uberaba - MG. A rocha fosfatada utilizada no processo de produção de ácido fosfórico era proveniente do Estado de Minas Gerais, mais especificamente, do município de Tapira, localizado a aproximadamente $400 \mathrm{~km}$ de Belo Horizonte. Após a coleta em cada um dos pontos de interesse, as amostras foram embaladas em sacos plásticos, os quais foram devidamente identificados e enviados para o laboratório.

Uma amostra composta de fosfogesso foi submetida à avaliação para classificação desse resíduo, segundo a Norma ABNT NBR 10004:2004 - Classificação Completa de Resíduos Sólidos, bem como à realização de análises da sua composição mineralógica pela técnica de difratometria de raios X (método do pó). Nesse caso, foi empregado um difratômetro de raios $\mathrm{X}$ de fabricação Rigaku, modelo DIMAX ÚLTIMA automático. A análise da composição química foi realizada pela técnica de fluorescência de raios $X$, sendo utilizado para isso, um espectrômetro de fluorescência de raios $X$ da marca SHIMADZU refrigerado a nitrogênio líquido.

A determinação da concentração de atividade dos radionuclídeos Ra-226, $\mathrm{Ra}-228$ e $\mathrm{Pb}-210$ foi realizada pelo método de espectrometria gama, do Th-232 por análise por ativação neutrônica e do ${ }^{238} \mathrm{U}$, por nêutrons retardados (OLIVEIRA, 2008). A medida da concentração dos elementos As e $\mathrm{Cr}$ presentes no fosfogesso foi feita por espectrometria de emissão atômica por plasma indutivamente acoplado e dos elementos $\mathrm{Hg}, \mathrm{Cd}$ e $\mathrm{Pb}$, por espectrofotometria de absorção atômica com forno de grafite.

\section{Determinação da proporção ideal da mistura resíduo sólido urbano/fosfogesso}

Após a caracterização do fosfogesso e obtenção dos resultados dos teores de radionuclídeos naturais e metais presentes no fosfogesso, a próxima etapa do trabalho constitui-se na determinação da proporção ideal da mistura resíduo sólido urbano/fosfogesso em condições de laboratório. Para tanto, foram construídas caixas de acrílico, nas dimensões $30 \times 30 \times 30 \mathrm{~cm}$, as quais foram usadas como reatores durante o procedimento experimental. Essas caixas foram totalmente seladas, possuindo apenas um orifício localizado na parte superior (tampa) e um na parte inferior (fundo da caixa). O orifício da parte superior foi conectado à entrada do detector utilizado para medida periódica das concentrações dos gases produzidos dentro de cada reator. No orifício da parte inferior foi conectada uma mangueira que permaneceu fechada até o momento da coleta do material lixiviado (chorume) produzido durante o processo de decomposição anaeróbica do resíduo.

Foram realizados quatro tratamentos nas seguintes proporções volumétrica de lixo: fosfogesso: 5:0, 5:1, 3:1 e 2:1 (T1, T2, T3 e T4), com três repetições, totalizando 12 unidades experimentais. A composição média do lixo utilizado em cada reator foi de $75 \%$ de material orgânico e $25 \%$ de material potencialmente reciclável (plástico e papel).

A mistura foi incubada anaerobicamente, de modo a simular a decomposição de resíduos sólidos em aterros sanitários. A quantidade ocupada pela mistura foi de aproximadamente $75 \%$ do volume total do "reator". Vale lembra que durante o processo anaeróbio pode formar, além de gás carbônico, metano e hidrogênio, outros compostos menos oxidados, tais como alcoóis ou ácidos orgânicos. Portanto, no presente estudo, o sistema de reatores permaneceu hermeticamente fechado durante todo o período de realização dos experimentos de forma a prevenir a contaminação e a perda dos gases produzidos durante a decomposição dos resíduos. Para todos os tratamentos foi necessário elevar a umidade inicial em $60 \%$ com o objetivo de acelerar a atividade biológica e de se ter umidade suficiente para a decomposição da matéria orgânica e, conseqüentemente, para a produção de chorume.

Antes do inicio dos experimentos foram coletadas amostras das misturas de cada unidade de tratamento. Essas amostras foram enviadas para o Laboratório de Matéria Orgânica da Universidade Federal de Viçosa, conservadas sob refrigeração a $4^{\circ} \mathrm{C}$, para posterior determinação da 
umidade, do pH e do teor de sólidos totais (ST) e sólidos totais voláteis (SV).

Após um período de aproximadamente 8 meses foi realizada a coleta do material lixiviado (chorume), o qual foi enviado para o laboratório para análise das concentrações de atividade radionuclídeos naturais e das concentrações de metais e metalóides. Neste momento, os reatores foram abertos e o material remanescente foi enviado para o Laboratório de Matéria Orgânica da Universidade Federal de Viçosa para se determinar novamente a umidade, do $\mathrm{pH}$ e o teor de sólidos totais (ST) e sólidos totais voláteis (SV).

O monitoramento da variação dos teores de ST e SV permitiu que, indiretamente, fosse feita uma avaliação do processo de aceleração da decomposição da matéria orgânica de cada amostra. A parte analítica para determinação dos parâmetros citados acima foi realizada utilizando-se os métodos preconizados pelo Standard Methods for Examination of Water and Wastewater (APHAAWWA-WPCF, 2005).

\section{Descrição da área de estudo}

Como a maior parte gerada de fosfogesso está disponibilizada no Estado de Minas Gerais, mais especificamente no município de Uberaba, e levando-se me conta a logística para o transporte de grandes quantidades desse material, necessárias para a operação diária de cobertura em grandes aterros (mais de 30.000 habitantes), o aterro municipal de Uberlândia foi escolhido como local de referência para a realização dos estudos de análise de segurança. Todas as informações descritas neste item foram retiradas do EIA/RIMA da expansão do Aterro Sanitário de Uberlândia, elaborado em 1996.

O volume total de resíduos coletados diariamente é de 26 toneladas. Os resíduos gerados em unidade de saúde são coletados separadamente, sendo esse resíduo reciclado. A separação do lixo é feita na usina de lixo e o chorume é depositado em tanques para decantação na própria usina de lixo. O composto extraído da usina é comercializado na região, como adubo. O restante do lixo, tais como papel, plástico, ferro, vidro e metais não ferrosos são vendidos para indústrias para serem reciclados novamente.

Todo o resíduo, após segregação, é acomodado em células, onde é colocada uma camada de material e a outra de terra, assim, até fechar os 4 metros de altura da célula. $\mathrm{O}$ lixo é disposto em camadas com cobertura diária sendo compactado por trator de esteiras. A camada de regularização e a camada superior são compostas por silte argiloso compactado, cujo coeficiente de permeabilidade $\mathrm{K}$ é menor que $1.10^{-5} \mathrm{~cm} / \mathrm{s}$, de acordo com o projeto do aterro. Além disso, a base do aterro é impermeabilizada com uma manta de polietileno de alta densidade (PEAD) com espessura de 2 $\mathrm{mm}$.

$\mathrm{O}$ aterro está implantado na vertente da margem direita do rio Uberabinha, na Bacia Sedimentar do Paraná, estando localizado numa porção onde os basaltos da formação Serra Geral constituem o substrato rochoso. O nível freático varia de 30 a $45 \mathrm{~m}$ de profundidade. $\mathrm{Na}$ área do Aterro de Uberlândia, os basaltos estão assentados diretamente sobre as rochas pré-cambrianas do Grupo Araxá e lentes de arenito da Formação Botucatu.

A presença do substrato basáltico subjacente aos materiais inconsolidados de textura argilo-arenosos (colúvio) ou silto-argiloso (residuais do basalto), com permeabilidade variando entre $2.10^{-5}$ e $7,8.10^{-5} \mathrm{~cm} / \mathrm{s}$ através de medidas in situ.

O rio Uberabinha é afluente do rio Araguari pela margem esquerda. Esse rio é responsável pelo abastecimento da população do município de Uberlândia que, segundo censo do IBGE Instituto Brasileiro de Geografia Estatística - possui uma população de 542.541 habitantes. $\mathrm{Na}$ Figura 1 é apresentada uma visão geral do aterro sanitário de Uberlândia e a localização do rio Uberabinha. 


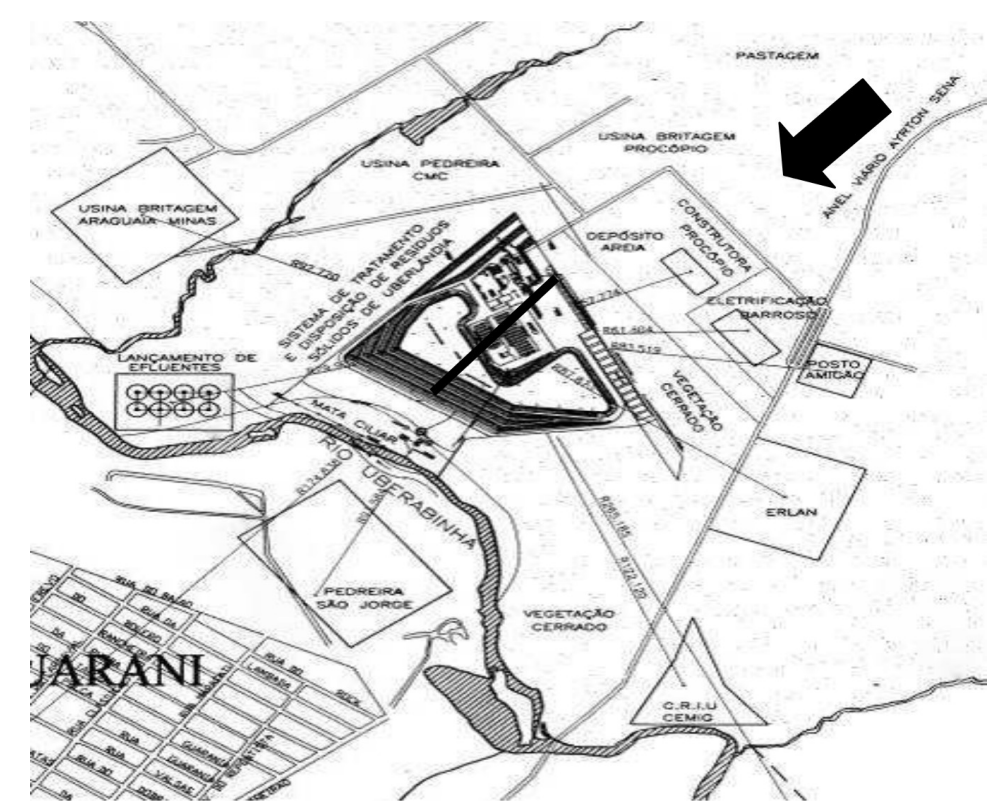

Figura 1 - Mapa de localização do aterro sanitário de Uberlândia; seta indicando o sentido preferencial do fluxo subterrâneo; linha indicando o perfil aproximado utilizado para o modelo no aquífero

Figure 1 - Location map of the Uberlândia sanitary landfill; arrow indicates the groundwater direction; line indicates the approximated simulated profile for the aquifer model

SIMULAÇÃO NUMÉRICA DOS PROCES- obtidos pelo EIA/RIMA), razão volumétrica da SOS DE FLUXO DE ÁGUA E TRANSPOR- mistura lixo e fosfogesso (razão ótima obtida TE DE CONTAMINANTES

\section{Inventário e termo-fonte}

O inventário de radionuclídeos e metais no aterro, associados à inclusão de fosfogesso como material de cobertura, foi estimado com base nos dados do aterro (volume de lixo e área, experimentalmente) e o teor medido desses contaminantes no fosfogesso. O inventário de radionuclídeos e metais no aterro, associados à inclusão de fosfogesso como material de cobertura é apresentado na Tabela 1 .

Tabela 1 - Inventário de radionuclídeos e metais no fosfogesso utilizado como material de cobertura do aterro Table 1 - Radionuclides and metais inventory of phosphogypsium

\begin{tabular}{ccc}
\hline Radionuclideo/metal & $\begin{array}{c}\text { Teor no fosfogesso } \\
(\mathrm{Bq} / \mathrm{kg} \text { ou } \mathrm{mg} / \mathrm{kg})\end{array}$ & $\begin{array}{c}\text { Massa ou atividade no } \\
\text { aterro } \\
\left(\mathrm{Bq} \mathrm{ou} \mathrm{mg} / \mathrm{cm}^{2}\right)\end{array}$ \\
\hline $\mathrm{U}-238$ & 40.0 & 67.5 \\
$\mathrm{U}-234$ & 40.0 & 67.5 \\
$\mathrm{Th}-230$ & 55.0 & 92.8 \\
$\mathrm{Ra}-226$ & 252.0 & 425.3 \\
$\mathrm{~Pb} 210$ & 206.0 & 347.6 \\
$\mathrm{Th}-232$ & 55.0 & 92.8 \\
$\mathrm{Ra}-228$ & 226.0 & 381.4 \\
$\mathrm{Ni}$ & 12.4 & 20.9 \\
$\mathrm{Cd}$ & 0.1 & 0.2 \\
$\mathrm{~Pb}$ & 20 & 33.8 \\
$\mathrm{As}$ & 3.4 & 5.7 \\
$\mathrm{Hg}$ & 0.6 & 1.0 \\
$\mathrm{Cr}$ & 38.2 & 64.5 \\
\hline \hline
\end{tabular}


A água de infiltração que sai da camada de lixo e entra na camada de drenos foi associada a uma concentração de cada um dos principais contaminantes analisados. Essa concentração foi estimada considerando que a taxa mássica de liberação dos contaminantes é proporcional ao inventário remanescente no termo-fonte a cada tempo, através da solução do sistema de equações 1a e $1 \mathrm{~b}$ (PONTEDEIRO, 2006):

$$
\frac{d M_{1}(t)}{d t}=-\gamma_{1} \cdot M_{1}(t)-\lambda_{1} \cdot M_{1}(t)
$$

$$
\underline{d M_{i}(t)}=-\gamma_{i} \cdot M_{i}(t)-\lambda_{i} \cdot M_{i}(t)+\lambda_{i^{-1}} \cdot M_{i^{-1}}(t) \text { para } i=2 \ldots n
$$

$\mathrm{dt}$

onde Mi é a massa ou atividade do elemento i por unidade de área da base do aterro $\left(\mathrm{g} / \mathrm{cm}^{2}\right) ; \gamma_{i}$ é a taxa de liberação do elemento i (1/dia); $\lambda_{1}$ é a taxa de decaimento radioativo do radionuclídeo i (1/dia), quando for o caso.

Considerando que a atividade remanescente no termo-fonte divide-se entre as fases sólida e aquosa e que a concentração na fase aquosa é dada pela isoterma de Freundlich aplicada ao caso linear, a taxa de liberação pode ser estimada por (PONTEDEIRO, 2006):

$$
\gamma_{i}=\frac{\operatorname{Inf}}{\left(\operatorname{Kd}_{A i \cdot} \rho_{b A}+\theta_{A}\right) \cdot H}
$$

onde Inf é a descarga específica através do aterro $\left(\mathrm{m}^{3} / \mathrm{m}^{2}\right.$.ano), $\mathrm{Kd}_{\mathrm{Ai}}$ é o coeficiente de distribuição do elemento i entre as fases sólida e aquosa den- tro do aterro $(\mathrm{ml} / \mathrm{g}), \rho_{\mathrm{bA}}$ é a densidade volumétrica do material dentro do aterro $(\mathrm{g} / \mathrm{ml}), \theta_{\mathrm{A}}$ é o conteúdo volumétrico de água no material do aterro $\left(\mathrm{m}^{3} / \mathrm{m}^{3}\right)$ e $\mathrm{H}$ é a espessura da camada de lixo $(\mathrm{m})$.

Em virtude da falta de dados específicos, para a estimativa da taxa de liberação de cada contaminante, foram utilizados valores de $\mathrm{Kd}$ considerando que o fosfogesso se comporta como um solo arenoso, em um cenário mais conservador, e como um solo argiloso, em um cenário mais realista.

\section{Modelagem numérica dos processos de fluxo e transporte}

Os processos de fluxo de água e transporte de contaminantes no aterro sanitário de Uberlândia foram simulados em duas etapas de modelagem, sendo abordados, separadamente, o fluxo predominantemente vertical das camadas inferiores do aterro e o fluxo predominantemente horizontal no aqüífero superficial.

\section{Modelos de fluxo e transporte nas camadas de fundo do aterro}

O modelo de fluxo das duas camadas do fundo do aterro sanitário de Uberlândia foi desenvolvido em elementos finitos através do programa HYDRUS 2D (versão 1.12), considerando uma seção transversal ao sentido preferencial do fluxo subterrâneo. As dimensões do modelo de fluxo estão descritas na Figura 2.

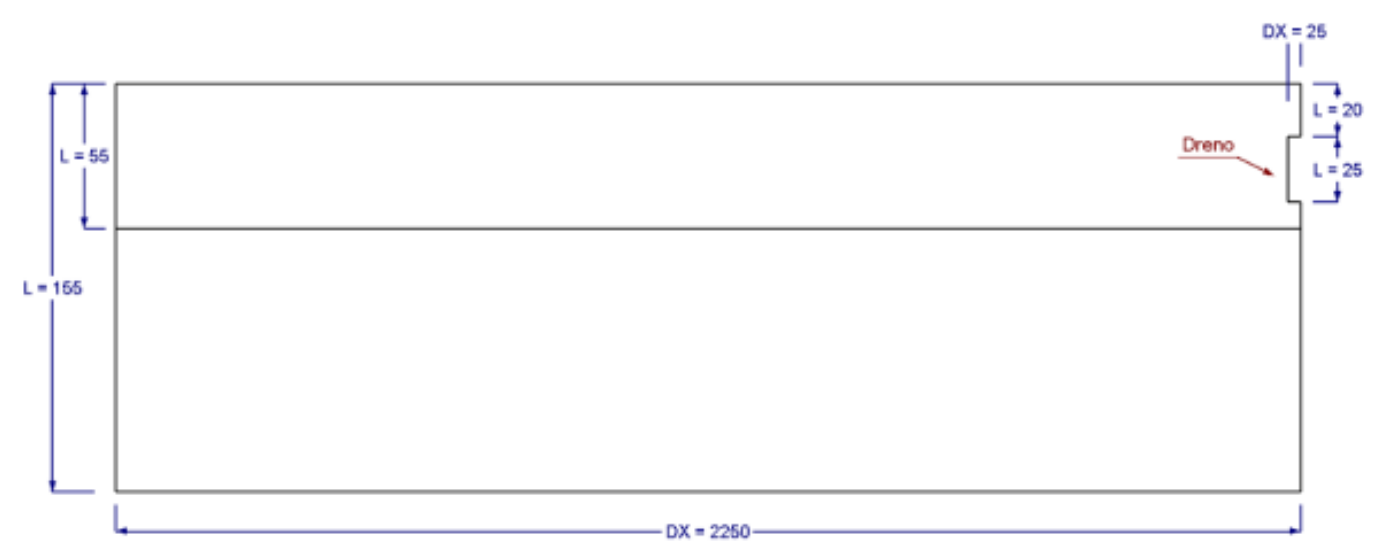

Figura 2 - Dimensões do modelo de fluxo do fundo do aterro sanitário de Uberlândia (valores em centímetros) Figure 2 - Dimensions of the flow model of the landfill bottom (values in centimeters) 
Ele é composto por duas camadas de diferentes materiais: a camada de drenos na parte superior, com $55 \mathrm{~cm}$ de espessura, e a barreira de argila no fundo, com $100 \mathrm{~cm}$ de espessura. Para a definição do comprimento horizontal foram considerados os divisores de água que os vários drenos dentro do aterro criam, tendo sido tomada a metade da distância entre dois drenos mais distantes $(2250 \mathrm{~cm})$. Na camada de drenos, um dos drenos foi incluído $(25 \mathrm{~cm}$ de altura, $50 \mathrm{~cm}$ de largura e localizado a $20 \mathrm{~cm}$ do topo da camada), representando o ponto de drenagem dessa camada. Como o problema é simétrico e visando um melhor desempenho computacional, apenas uma das metades foi simulada. Nesse caso, uma malha uniforme foi criada com elementos triangulares de $5 \mathrm{~cm}$ de lado.

Dois tipos de materiais foram atribuídos aos nódulos da malha de elementos finitos, representando as propriedades de um material altamente permeável (areia), constituindo a camada superior dos drenos, e um material de baixa permeabilidade (argila), na camada inferior. $\mathrm{Na} \mathrm{Ta-}$ bela 2 são apresentadas as propriedades hidráulicas utilizadas para cada material, sendo que, em geral, os valores assumidos para os coeficientes do modelo de fluxo não-saturado de van Genuchten-Mualem foram obtidos na literatura (PONTEDEIRO, 2006), com exceção da condutividade hidráulica saturada da camada de argila, que foi obtida dos dados do EIA/RIMA do aterro.

O modelo foi construído para simulação do fluxo de água e transporte de contaminantes em regime transiente. Para as condições iniciais do modelo, a pressão da água foi assumida como sendo nula (condição de saturação) na camada de argila e em equilíbrio hidrostático na camada de drenos. As condições de contorno foram mantidas invariáveis ao longo de todo o período de simulação. No topo do modelo (Figura 3), a condição de contorno de fluxo especificado descreve a água infiltrada a partir da precipitação, sendo utilizado o valor de $0,2 \mathrm{~cm} / \mathrm{dia}$ (730 mm/ano). Esse valor foi estimado através das médias do excedente hídrico obtido pelo balanço hídrico (THORNTHWAITE \& MATHER, 1985) com dados de estação meteorológica de Uberlândia entre 2003 e 2009 (banco de dados Instituto Nacional de Meteorologia - INMET).

Em torno do dreno, duas condições de contorno (Figura 3) foram assinaladas: fluxo nulo para a metade inferior do dreno e face de percolação na metade superior (saída de água à pressão atmosférica ou "seepage face"). Essas duas condições de contorno combinadas refletem a consideração de eficiência parcial da remoção de água pelos drenos (nível da água dentro dos drenos em sua meia-altura), que é uma situação mais conservadora. No fundo, foi considerado que a pressão é mantida nula, refletindo a mudança da condição de saturação da camada de argila para uma condição parcialmente saturada do solo natural. A simulação de fluxo de água foi desenvolvida em regime transiente até se atingir a condição permanente, uma vez que não foi possível obter a solução direta para o fluxo permanente. Para a simulação transiente foi assumida uma tolerância de 0,001 para o conteúdo de água e de $0,1 \mathrm{~cm}$ para a carga hidráulica de pressão.

Tabela 2 - Parâmetros de Van Genuchten de cada material do fundo do aterro sanitário de Uberlândia Table 2 - Van Genuchten properties for each material of the Uberlândia`s landfill bottom layer

\begin{tabular}{ccccccc}
\hline Material & $\begin{array}{c}\theta_{\mathrm{r}} \\
(-)\end{array}$ & $\begin{array}{c}\theta_{\mathrm{s}} \\
(-)\end{array}$ & $\begin{array}{c}\alpha \\
(1 / \mathrm{cm})\end{array}$ & $\begin{array}{c}\mathrm{n} \\
(-)\end{array}$ & $\mathrm{K}_{\mathrm{s}}(\mathrm{cm} / \mathrm{dia})$ & $\begin{array}{c}1 \\
(-)\end{array}$ \\
\hline 1 (areia) & 0,045 & 0,3 & 0,145 & 2,68 & 712,8 & 0,5 \\
2 (argila) & 0 & 0,06 & 0,00131 & 1,048 & 0,086 & 0,5 \\
\hline
\end{tabular}




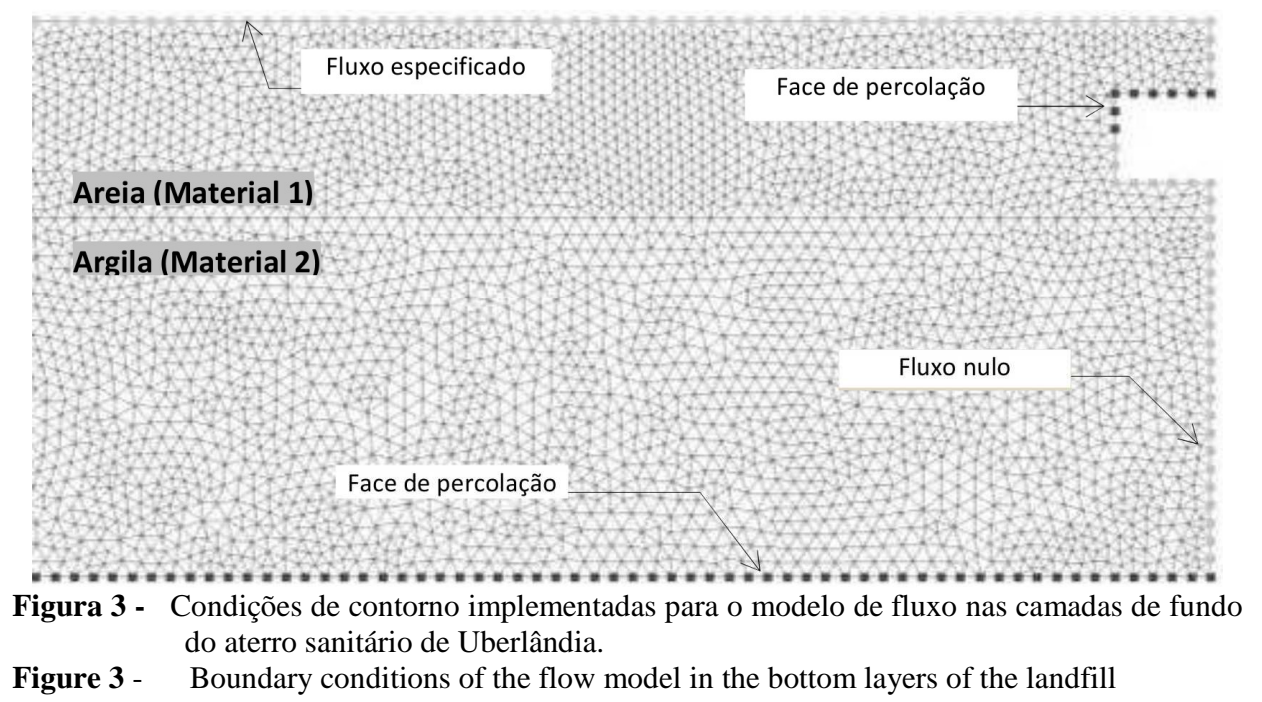

Para a simulação de transporte de contaminantes no fundo do aterro sanitário foi construído um modelo unidimensional em elementos finitos (HYDRUS) composto somente da camada de argila, por ser a camada em que o principal mecanismo de retardo ocorre. Nesse modelo, a distribuição da pressão da água reproduz os resultados obtidos no modelo bidimensional de fluxo (regime estacionário), sendo considerada a coluna com a maior carga hidráulica (coluna mais distante do dreno). O objetivo da aplicação desse modelo foi obter um ganho de eficiência computacional durante a simulação de transporte de contaminantes, que é computacionalmente mais exigente. Para garantir melhores resultados em termos de redução de oscilação numérica e dispersão artificial, o valor do elemento finito triangular utilizado foi de $1 \mathrm{~cm}$, que, juntamente com o valor de dispersividade de $1 \mathrm{~cm}$, levam a um valor do número de Peclet igual a 1 .

Com relação às propriedades relacionadas à simulação de transporte, a densidade volumétrica da argila foi considerada como sendo $1,93 \mathrm{~g} / \mathrm{cm}^{3}$, de acordo com a porosidade total sugerida no RESRAD (YU et al., 1993) para a argila (42\%). A dispersividade foi assumida como sendo $1 \mathrm{~cm}$, seguindo o critério de utilizar entre 1/100 e 1/10 do valor da distância a ser percorrida.

Duas reações foram consideradas no modelo de transporte: adsorção e decaimento radioativo (quando for o caso). Para os dados de porosidade total e efetiva para a argila foram tomados os valores de referência para a argila do manual para obtenção de dados para o código RESRAD, utilizado para análise de segurança de instalações radiativas e nucleares. Valores de coeficiente de adsorção $(\mathrm{Kd})$ foram utilizados para descrever a reação de adsorção segundo a isoterma linear de Freundlich (USEPA, 2005) para os contaminantes simulados (adsorção simulada apenas para a camada de argila), e os coeficientes de decaimento radioativo são usados para simular as cadeias de decaimento.

Os valores de Kd utilizados foram escolhidos com base nos valores recomendados para a argila no manual para obtenção de dados para o código RESRAD. O manual inclui valores default de Kd para alguns elementos baseado em diferentes compilações de literatura e no estudo de Sheppard e Thibault (1955). Não existem valores de Kd para o arsênio e mercúrio nas tabelas orientativas do RESRAD. Nesse caso, utilizou-se o valor mediano da compilação da U.S.EPA de valores de Kd para metais (USEPA, 2005), conforme apresentado na Tabela 3. 
Tabela 3 - Valores de Kd para a simulação de transporte na camada de argila e no aquífero Table 3 - Kd values for transport simulation for the clay layer and aquifer

\begin{tabular}{cc}
\hline \hline Elemento & $\mathrm{Kd}\left(\mathrm{cm}^{3} / \mathrm{g}\right)$ \\
\hline $\mathrm{U}$ & 1600 \\
$\mathrm{Th}$ & 5800 \\
$\mathrm{Ra}$ & 9100 \\
$\mathrm{~Pb}$ & 550 \\
$\mathrm{Ni}$ & 650 \\
$\mathrm{Cd}$ & 560 \\
$\mathrm{Hg}$ & 6300 \\
$\mathrm{Cr}$ & 1500 \\
$\mathrm{As}$ & 2500 \\
\hline \hline
\end{tabular}

Fonte: RESRAD para argila e USEPA (2005)

O modelo de transporte foi construído para simulação em regime transiente. Para as condições iniciais do modelo, a concentração dos solutos foi considerada nula em todos os elementos do modelo. No topo da coluna foi aplicada a condição de terceiro tipo, em que a concentração é especificada para o topo do modelo e a taxa mássica de entrada reflete essa concentração e a taxa de água que é admitida no sistema. A evolução da concentração ao longo no topo da coluna de argila foi estimada por meio da análise da depleção dos contaminantes no termo-fonte, por decaimento radioativo e por lixiviação conforme especificado por (PONTEDEIRO, 2006).

\section{Modelo de fluxo e transporte no aqüífero}

Para a simulação de fluxo e transporte de contaminantes na zona saturada do aquífero diretamente sob influência do aterro sanitário, um modelo bidimensional foi construído, representando um corte transversal paralelo à direção preferencial de fluxo do aquíf́ero.
Para a construção do modelo foi utilizado os modelos em diferenças finitas MODFLOW (HARBAUGH, 2005) e RT3D (CLEMENT, 1997), através do programa Visual MODFLOW (versão 4.3). A seta na Figura 1 indica o sentido preferencial do fluxo subterrâneo assumido.

As dimensões do modelo estão mostradas na Figura 4. O modelo abarca toda a área do aterro sanitário $(370 \mathrm{~m})$ e se estende até o rio Uberabinha, localizado a $500 \mathrm{~m}$ do início do modelo. A superfície do terreno apresenta duas inclinações diferentes, uma área mais plana correspondendo à área do aterro (inclinação de $\sim 0,6 \%$, entre as cotas 810 e 789 m) e outra mais íngreme, correspondente à faixa de terreno natural desde o fim do aterro até a margem do rio (inclinação de $30 \%$, entre as cotas 789 e $750 \mathrm{~m}$ ). O limite impermeável inferior do modelo foi fixado na cota $740 \mathrm{~m}$. A malha em diferenças finitas criada para o domínio é composta por 100 colunas (espaçamento de $5 \mathrm{~cm}$ ) e quatro camadas, como pode ser isto visto na Figura 5.

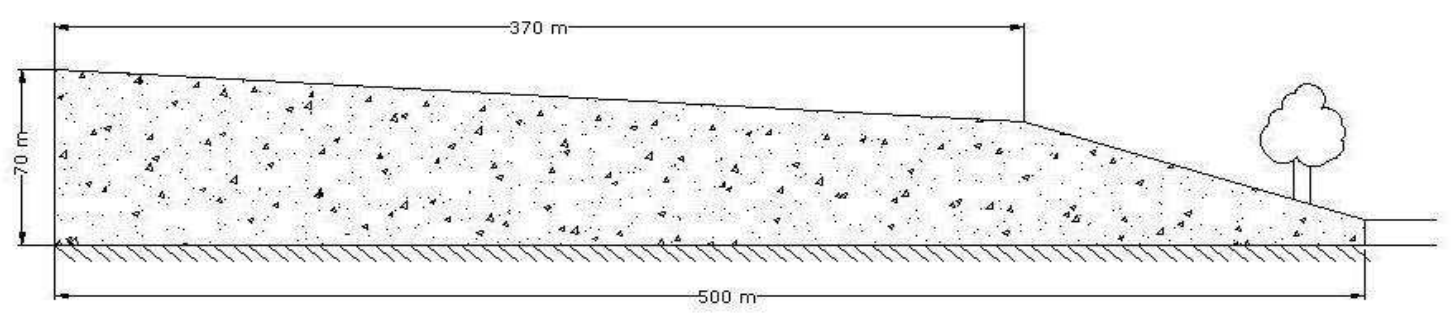

Figura 4 - Dimensões do domínio do modelo do aqüífero abaixo do aterro sanitário de Uberlândia Figure 4 - Dimensions of the aquifer model bellow the landfill 
Conforme mencionado anteriormente, para esse modelo o aqüífero foi considerado homogêneo e isotrópico, composto de material arenoso-argilo com condutividade hidráulica igual a $7,8.10^{-4} \mathrm{~cm} / \mathrm{s}$, porosidade total igual a $30 \% \mathrm{e}$ porosidade efetiva igual a $25 \%$, escolhidos com base em uma leitura conservadora dos dados do
EIA/RIMA. Um gradiente hidráulico de aproximadamente $10 \%$ foi simulado impondo condições de contorno tipo carga especificada nas duas extremidades do domínio (início do aterro e rio Uberabinha), com valores iguais a 802 e $750 \mathrm{~m}$, respectivamente, conforme é mostrado na Figura 5.

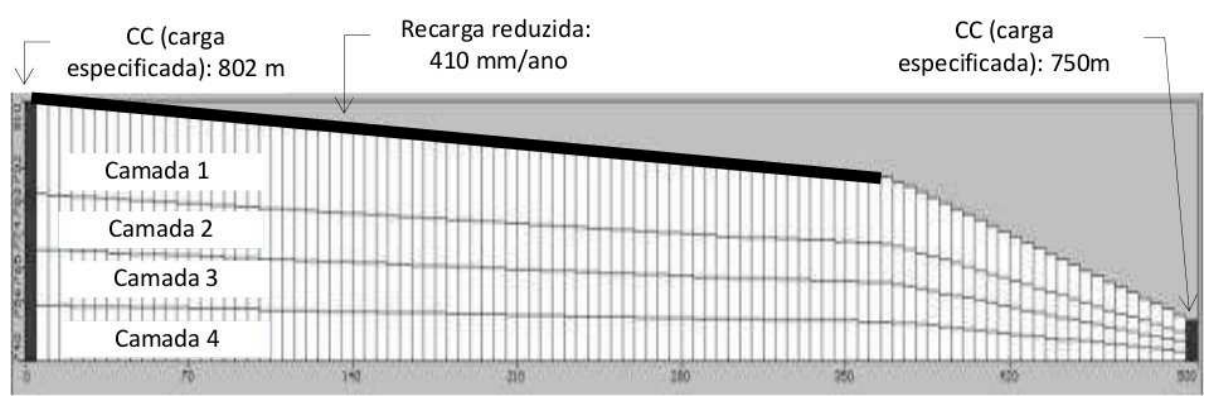

Figura 5 - Malha e condições de contorno associadas ao modelo do aqǘf́ero abaixo do aterro sanitário de Uberlândia

Figure 5 - Grid and boundary conditions for the model of the aquifer bellow the landfill

Além dessa condição de contorno de fluxo, também foi acrescentado o aporte proveniente da recarga superficial: $410 \mathrm{~mm} / \mathrm{ano}$ referente à recarga sob o aterro sanitário, definido pelo resultado do modelo de fluxo não-saturado nas camadas de fundo do aterro. A recarga no trecho entre $o$ aterro e o rio foi suprimida por se tratar de uma declividade acentuada, que reduz a recarga local. Todas as condições de contorno foram mantidas inalteradas ao longo de todo o período de simulação. Para as condições iniciais do modelo, a carga hidráulica foi assumida como sendo $810 \mathrm{~m}$ em todo o domínio e o modelo de fluxo foi executado em modo permanente.

Com relação ao transporte de contaminantes, uma concentração nula foi assumida para todos os contaminantes simulados no domínio inteiro no tempo inicial e o modelo de transporte foi executado em modo transiente. Foi estabelecida uma condição de terceiro tipo para o topo do modelo, em que a concentração é especificada e a taxa mássica de entrada reflete essa concentração e a taxa volumétrica de água que é admitida no sistema. A evolução temporal da concentração dos contaminantes foi estabelecida pela concentração de saída do modelo unidimensional de transporte na camada de argila. Para as propriedades relacionadas à simulação de transporte, foi assumida uma densidade volumétrica de
$1,855 \mathrm{~g} / \mathrm{cm}^{3}$ e dispersividades longitudinal e transversal de 5 e $0,5 \mathrm{~cm}$, respectivamente, equivalente à $10 \%$ da distância a ser percorrida pela pluma (500 metros) no eixo longitudinal e a 10\% deste valor para o eixo transversal.

Os mesmos contaminantes e cadeias de decaimento simulados no modelo das camadas de fundo do aterro sanitário também são simulados neste caso, bem como o uso da reação de adsorção segundo a isoterma linear de Freundlich para os contaminantes simulados no modelo do aqüífero. Os valores de $\mathrm{Kd}$ foram os mesmos utilizados para a camada de argila, uma vez que o aquífero tem forte caráter argiloso (Tabela 3).

\section{RESULTADOS E DISCUSSÕES}

\section{Caracterização do resíduo fosfogesso e deter- minação da fração ideal de mistura lixo urba- no: fosfogesso}

O resíduo fosfogesso foi classificado como Classe IIA - Não perigoso, Não Inerte, Não Corrosivo e Não Reativo. $\mathrm{O}$ pH da amostra foi de $4,22 \pm 0,04$. Portanto, dentro do intervalo aceito pela Norma ABNT NBR 10.004:2010, o qual não pode ser inferior a 2,0 ou superior a 12,5.

Os resultados das análises para caracterização química do fosfogesso revelaram a presença predominante de cálcio e enxofre. Em média, a quantidade de $\mathrm{P}_{2} \mathrm{O}_{5}$ encontrada é da 
ordem de 1,2\%. Os resultados das análises mineralógicas da amostra de fosfogesso indicam que ele é constituído essencialmente de Gipso $\left(\mathrm{CaSO}_{4} \cdot 2 \mathrm{H}_{2} \mathrm{O}\right)$; teores traços de Anidrita $\left(\mathrm{CaSO}_{4}\right)$, Bassanita $\left(\mathrm{CaSO}_{4} \cdot 0,5 \mathrm{H}_{2} \mathrm{O}\right)$, Quartzo $\left(\mathrm{SiO}_{2}\right)$ e Muscovita $\mathrm{Al}_{2}\left(\mathrm{AlSi}_{3} \mathrm{O}_{10}\right)(\mathrm{OH})_{2}$.

$\mathrm{Na}$ Tabela 4 são apresentados os resultados da concentração de cada um dos radionuclídeos e metais analisados na amostra de fosfogesso utilizada no presente estudo. Como pode ser observado, a concentração de atividade média do ${ }^{238} \mathrm{U}$ no fosfogesso $\left(80 \mathrm{~Bq} \cdot \mathrm{kg}^{-1}\right)$ ficou abaixo daquela do ${ }^{232} \mathrm{Th}\left(111 \mathrm{~Bq} \mathrm{~kg}^{-1}\right)$. Isso pode ser explicado pelo fato da rocha fosfatada utilizada para a produção de ácido fosfórico ser de origem ígnea e, portanto, apresenta concentrações de $\mathrm{U}$ menores que as de Th.

Tabela 4 - Concentração de radionuclídeos naturais e metais presentes no fosfogesso

Table 4 - Natural radionuclides and metals concentration in phosphogypsium

\begin{tabular}{cccc}
\hline Radionuclídeo & $\begin{array}{c}\text { Concentração } \\
\left(\mathrm{Bq}^{-k g^{-1}}\right)\end{array}$ & Metais & $\begin{array}{c}\text { Concentração } \\
\left(\mathrm{mg} \mathrm{kg}^{-1}\right)\end{array}$ \\
\hline${ }^{238} \mathrm{U}$ & $80 \pm 20$ & $\mathrm{As}$ & $3,4 \pm 0,1$ \\
${ }^{232} \mathrm{Th}$ & $111 \pm 13$ & $\mathrm{Cd}$ & $<0,1$ \\
${ }^{226} \mathrm{Ra}$ & $252 \pm 26$ & $\mathrm{Cr}$ & $38,2 \pm 0,1$ \\
${ }^{228} \mathrm{Ra}$ & $226 \pm 29$ & $\mathrm{Hg}$ & $<0,025$ \\
${ }^{210} \mathrm{~Pb}$ & $206 \pm 29$ & $\mathrm{~Pb}$ & $<20$ \\
\hline
\end{tabular}

Os resultados dos experimentos de biodegradação anaeróbica dos resíduos sólidos urbanos em laboratório demonstraram que a amostra de chorume gerada pela mistura de resíduo sólido urbano/fosfogesso numa proporção de 3:1 foi a que apresentou menores valores de concentração de radionuclídeos e metais, inclusive que a amostra gerada pela mistura de resíduo sólido urbano/fosfogesso numa proporção de 1:1, ou seja, sem fosfogesso.

Além disto, foi observado que a variação no teor de sólidos totais voláteis $(37,6 \%)$ foi maior no reator que continha a mistura de resíduo sólido urbano/fosfogesso na proporção de 3:, o que indicou que essa foi a amostra que apresentou maior percentual de decomposição de matéria orgânica durante o intervalo de tempo de realização dos experimentos (JACOMINO et al., 2011).

\section{Modelo de fluxo de água e transporte nas camadas de fundo do aterro}

A Figura 6 apresenta a evolução temporal da concentração de alguns dos contaminantes (série do U-238) presentes no termo-fonte, considerando as taxas de liberação estimada para $\mathrm{o}$ aterro contendo fosfogesso. Embora a análise tenha sido feita até 10.000 anos, o termo-fonte se mostrou esgotado após um período de 1.000 anos. 


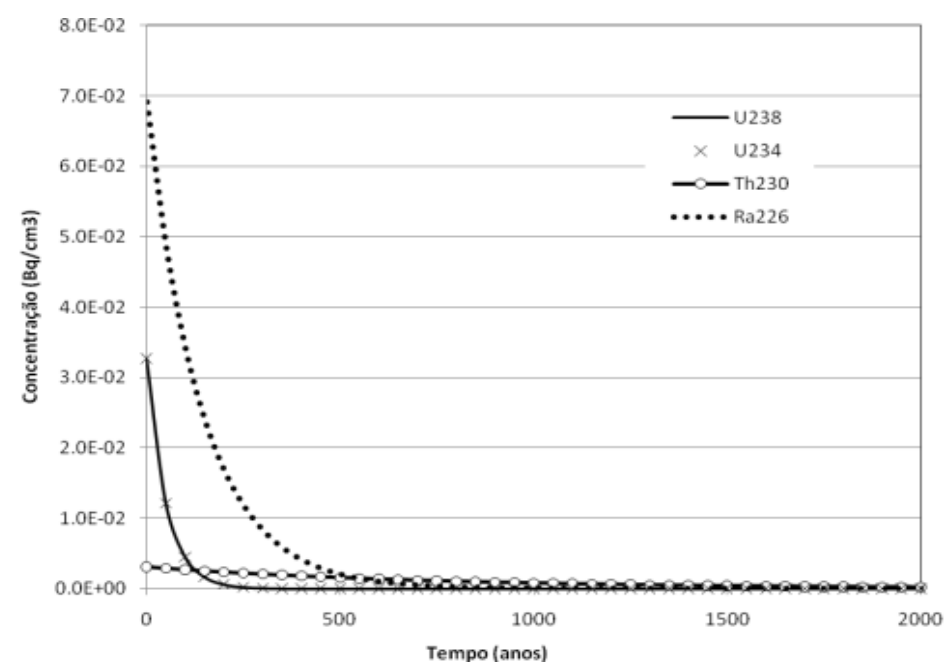

Figura 6 - Evolução da concentração dos contaminantes no termo-fonte (série do U- 238)

Figure 6 - Evolution of the contaminant concentrations in the source-term (U-238 serie)

Na Figura 7 é apresentada a distribuição de carga hidráulica de pressão inicial e a sua evolução no domínio para 100 dias de simulação, respectivamente. A linha amarela indica, aproximadamente, a posição da superfície potenciomé- trica $(\mathrm{h}=0)$. A análise dos resultados final no domínio sugeriu que, para fins práticos, o regime de fluxo permanente foi estabelecido após 100 dias de simulação.
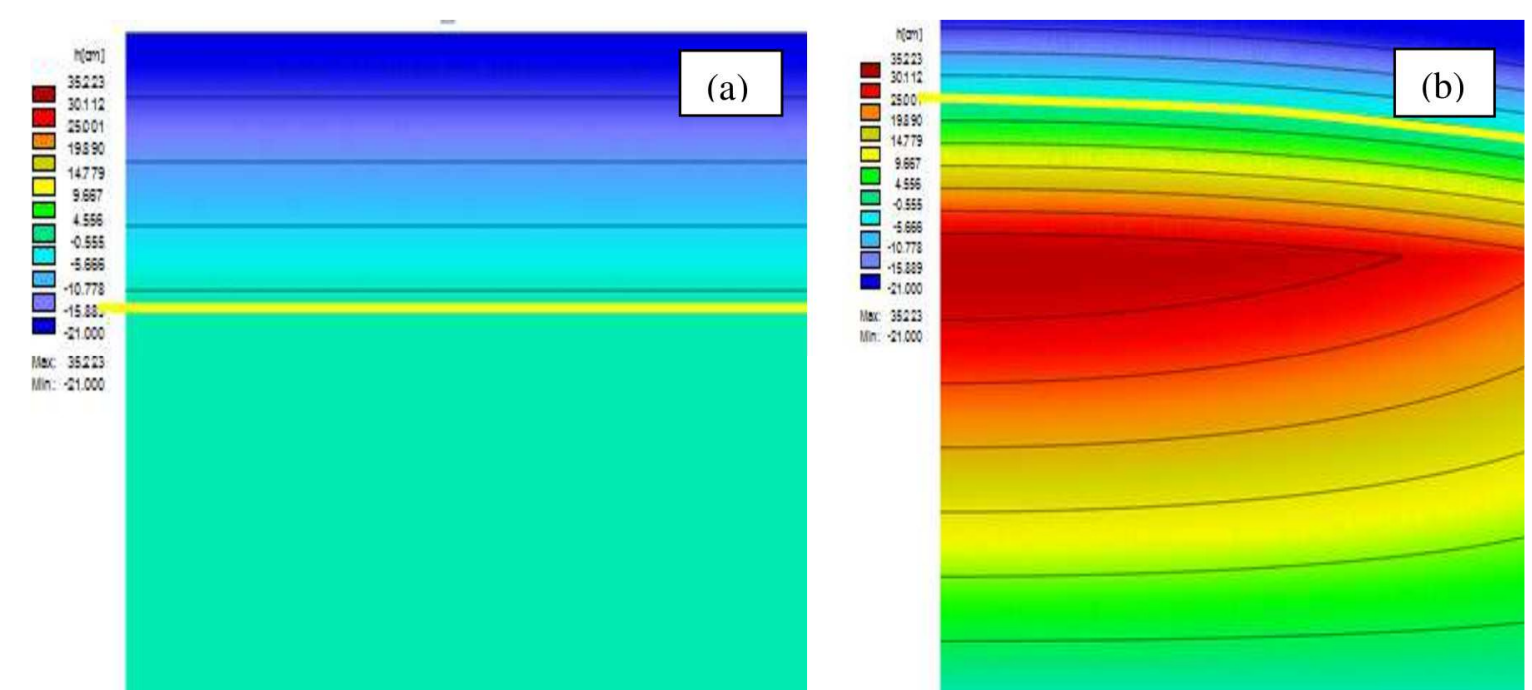

Figura 7 - Distribuição de carga hidráulica no modelo de fluxo de água no fundo do aterro sanitário: (a) condição inicial e (b) 100 dias

Figure 7 - Hydraulic head distribution in the landfill bottom flow model: (a) initial condition and (b) 100 days

O tempo de residência da água captada pelo dreno variou de 50 a 70 dias, para a linha de fluxo mais próxima e para a mais distante do dreno, respectivamente.

Considerando a linha de fluxo mais afastada do dreno que atravessa a camada de argila, o tempo de percurso até a interface com a argila e até o fundo dessa camada foi de 90 e 325 dias, respectivamente. A velocidade máxima através da argila pode ser estimada, portanto, como sendo $0,43 \mathrm{~cm} /$ dia.

O modelo unidimensional da camada de argila desenvolvido para a simulação dos processos de transporte de contaminantes dissolvidos nessa camada foi também implementado com sucesso. A solução de fluxo em regime permanente foi atingida, resultando na mesma distribuição de pressões encontrada para o modelo bidi- 
mensional, considerando a coluna mais afastada do dreno.

Como previsto através das linhas de fluxo e da estimativa de tempo de residência de partículas no campo de fluxo, a pluma deixou o domínio através de seu contorno inferior após 300 dias.

Essa situação pode ser considerada verdadeira para um sistema conservativo, sem a atuação de reações de adsorção, que promovam o atraso da pluma (retardo), nem de decaimento radioativo. Considerando o efeito de retardo na camada de argila equivalente a um valor de $\mathrm{Kd}$ igual a $10 \mathrm{~cm}^{3} / \mathrm{g}$, o efeito seria atrasar o avanço da pluma em 71 vezes, fazendo com que a velocidade de avanço desse contaminante hipotético seja reduzida para $5,93 \cdot 10^{-3} \mathrm{~cm} / \mathrm{dia}$ na argila.

\section{Modelo de fluxo de água e transporte de con- taminantes no aquífero}

A Figura 8 mostra o resultado da simulação de fluxo em regime permanente do aquífero abaixo do aterro sanitário, gerado pelo programa MODFLOW. Nessa figura é apresentada a linha piezométrica que delimita a zona saturada do aqüífero, as isolinhas de carga hidráulica e setas indicativas da direção do fluxo para o perfil do solo simulado.

O resultado mostra o fluxo subsuperficial do aquífero sendo descarregado no rio Uberabinha, estando de acordo com o modelo conceitual de fluxo especificado para a área e implementado no modelo de fluxo. Para os metais, a simulação foi realizada apenas para o $\mathrm{Ni}, \mathrm{Pb}, \mathrm{As}$ e $\mathrm{Cr}$, já que a simulação de transporte na camada de argila resultou em concentrações muito pequenas para o $\mathrm{Hg}$ e Cd na interface com o aquífero.As concentrações máximas obtidas nos pontos de observação, ao longo dos 10.000 anos analisados, são mostradas na Tabela 5 para os radionuclídeos e para os metais. Os pontos de observação considerados foram o final do aterro (Ponto A), logo abaixo da camada de argila, e o rio Uberabinha (Ponto B), na primera camada do modelo.

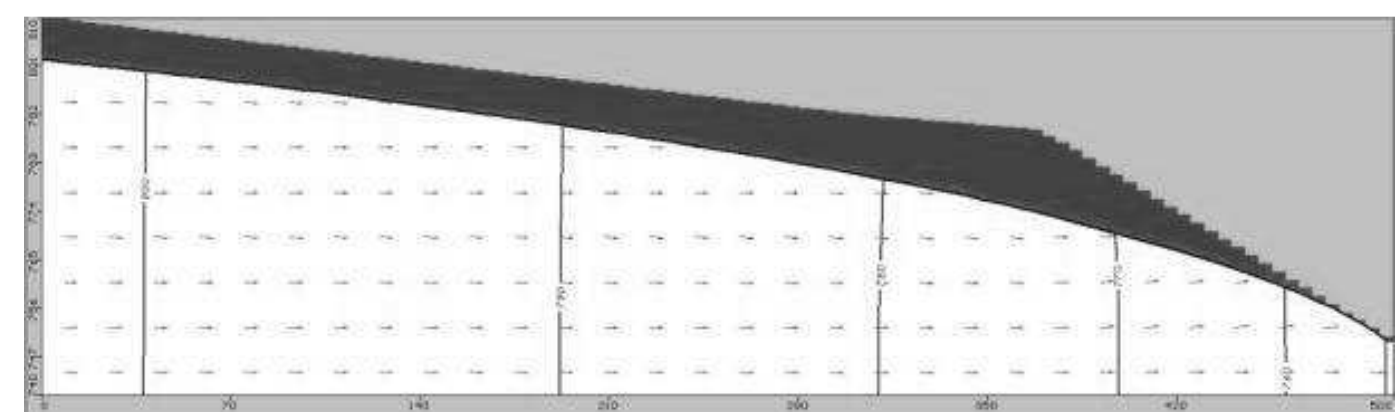

Figura 8 - Distribuição de cargas hidráulicas e vetores indicativos de direção de fluxo no modelo de fluxo do aqüífero (valores em metros)

Figure 8 - Hydraulic head distribution and flow direction vectors in the aquifer model (values in meters)

Uma vez que os resultados para metais apresentaram-se acima do limite de potabilidade estabelecido Ministério da Saúde (2004), novas simulações foram realizadas assumindo uma condição mais realística em que o fosfogesso possui um comportamento muito mais semelhante aos dos solos argilosos do que arenosos (OLIVEIRA, 2008). Neste caso, foram utilizados valores de $\mathrm{Kd}$ associados à taxa de liberação dos contaminantes no fosfogesso semelhantes ao de um solo argiloso (vide Tabela 3). 
Tabela 5 - Concentrações máximas obtidas nos pontos de observação, após 10.000 anos de simulação

Table 5 - Maximum concentration in observation points, after 10,000 years of simulation

\begin{tabular}{cccc}
\hline \hline Radionuclídeo & $\begin{array}{c}\text { Concentração } \\
\text { máxima (Ponto } \\
\text { A) (Bq/L) }\end{array}$ & $\begin{array}{c}\text { Concentração } \\
\text { máxima (Ponto } \\
\text { B) (Bq/L) }\end{array}$ & $\begin{array}{c}\text { Limites de } \\
\text { Potabilidade } \\
(\mathrm{Bq} / \mathrm{L})\end{array}$ \\
\hline $\mathrm{U}-238$ & 1.322 & 0.738 & 10 \\
$\mathrm{U}-234$ & 1.017 & 0.735 & 1 \\
$\mathrm{Th}-230$ & 0.146 & 0.082 & 1 \\
$\mathrm{Ra}-226$ & 0.257 & 0.144 & 1 \\
$\mathrm{Th}-232$ & 0.032 & $<10^{-8}$ & 1 \\
$\mathrm{Ra}-228$ & $\sim 0$ & $\sim 0$ & 0,1 \\
\hline \hline & Concentração & Concentração & Limites de \\
Elemento & máxima (Ponto & máxima (Ponto & Potabilidade \\
& $\mathrm{A})(\mathrm{mg} / \mathrm{L})$ & $\mathrm{B})(\mathrm{mg} / \mathrm{L})$ & 0,02 \\
$\mathrm{Ni}$ & 0.070 & 0.023 & 0,01 \\
$\mathrm{~Pb}$ & 0.149 & 0.059 & 0,01 \\
$\mathrm{As}$ & 0.067 & 0.049 & 0,05 \\
$\mathrm{Cr}$ & 0.722 & 0.428 & \\
\hline $\mathrm{A}$ & & & \\
\hline
\end{tabular}

Ponto A: final do aterro, logo abaixo da camada de argil

Ponto B: Rio Uberabinha, na primeira camada do modelo

Na Figura 9 é apresentada a variação entre as camadas de lixo misturado com fosfotemporal da concentração dos metais na interface gesso e a barreira da argila é bastante lenta, aprelixo/fosfogesso e barreira de argila considerando sentando comportamento linear. Nesse caso, os que o fosfogesso apresente os mesmos valores de valores de concentração ficaram abaixo dos limiKd dos solos argilosos. Conforme pode ser veri- tes de potabilidade estabelecidos pelo Ministério ficado, a taxa de liberação dos metais na interface da Saúde (BRASIL, 2004).

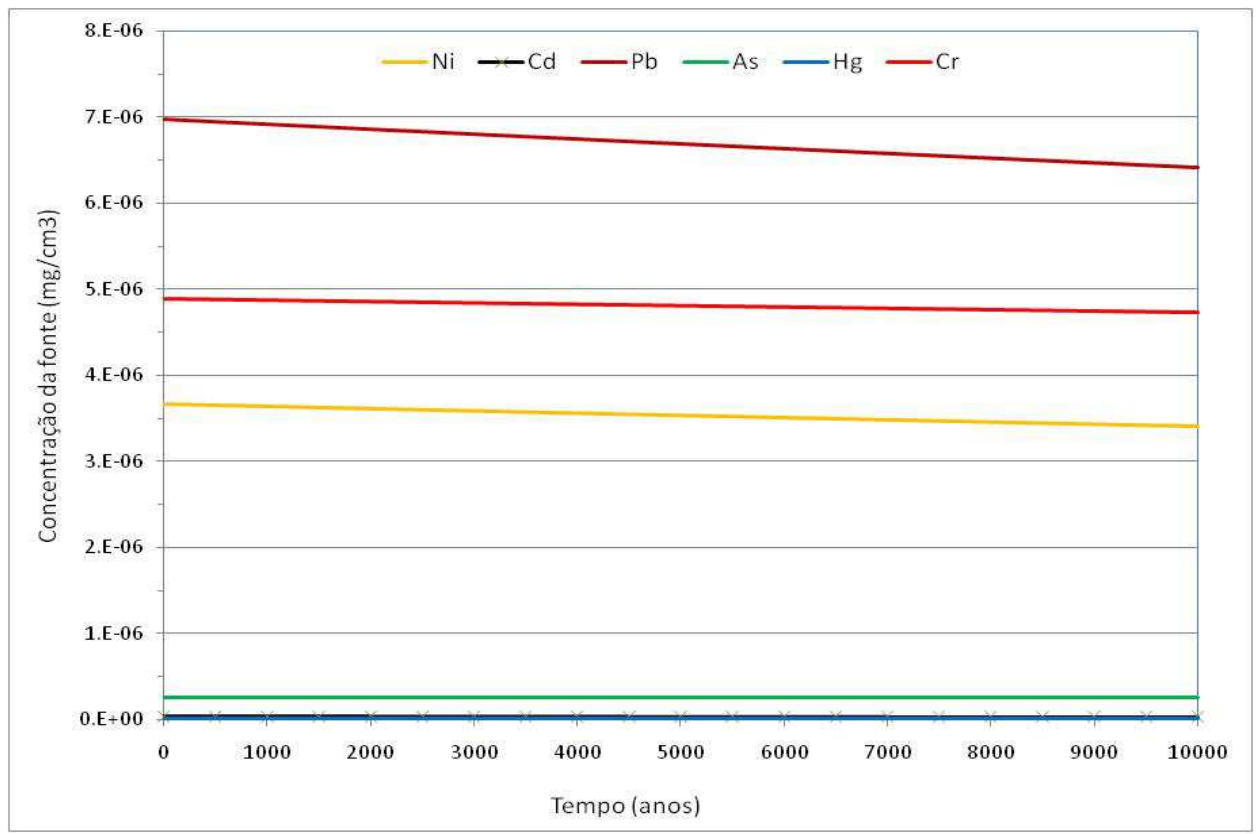

Figura 9 - Evolução da concentração dos metais na interface lixo/fosfogesso e barreira de argila considerando Kd de solo argiloso

Figure 9 - Evolution of metals concentration at the waste/phosphogypsium and clay layer interface considering Kd for clayer soil 


\section{CONCLUSÕES}

Os resultados da modelagem matemática nos pontos de observação aprontaram concentrações (em termos de atividade) dos radionuclídeos analisados abaixo dos limites de potabilidade estabelecidos pela legislação (WHO, 2008). Com relação aos metais, alguns resultados, em especial o do chumbo, ficaram acima do limite de potabilidade recomendado pelo Ministério da Saúde (BRASIL, 2004) para o cenário mais conservador em que se assume que os valores do coeficiente de distribuição $(\mathrm{Kd})$ do fosfogesso eram semelhantes ao de um solo arenoso.

Utilizando o cenário em que o fosfogesso possui um comportamento mais aproximado aos de solos argilosos do que de arenosos, todos os resultados de concentração máxima dos elementos de interesse, radioativos ou não, obti dos nos pontos de observação considerados ficaram abaixo do limite de potabilidade recomendado pelo Ministério da Saúde (2004).

$\mathrm{O}$ estudo realizado permitiu concluir que, mesmo no cenário mais conservador de liberação dos contaminantes pelo fosfogesso, a aplicação do fosfogesso como material de cobertura de aterros sanitários não resulta em risco à saúde humana e ao meio ambiente devido aos radionuclídeos presentes.

Foi possível perceber a necessidade de um estudo mais aprofundado sobre o comportamento de liberação dos contaminantes pelo fosfogesso. Considerando um comportamento mais realista, constatou-se que os metais presentes no fosfogesso também não acarretam risco pelo uso proposto desse material.

\section{AGRADECIMENTOS}

Os autores gostariam de agradecer à Fundação de Amparo à Pesquisa do Estado de Minas Gerais (FAPEMIG) pelo financiamento deste projeto.

\section{REFERÊNCIAS}

APHA, AWWA, WEF. Standard methods for the examination of water and wastewater. Washington,DC, $21^{\text {th }} \mathrm{Ed}$; 2005.

ASSOCIAÇÃO BRASILEIRA DE NORMAS. Resíduos sólidos: classificação. Rio de Janeiro, 2010. 71 p. ABNT NBR 10004:2010.

BIRDSELL, K.H.; WOLFSBERG, A.V.; HOLLIS, D. Groundwater flow and radionuclide transport calculations for a performance assessment of a low level waste site. Journal of Contaminant Hydrology, v. 46, p. 99 - 129, 2000.

BRASIL. Ministério da Saúde. Portaria no 518 de 25 de março de 2004.Estabelece normas e o padrão de potabilidade da agua destinada ao consumo humano. Diário Oficial da União, Brasília, v. 59, p. 226 - 270, 26 mar. 2004, Seção 1.

CANUT, M. Estudo da viabilidade da substituição do gesso pelo resíduo fosfogesso em material de construção. Belo Horizonte, $110 \mathrm{f}$. Dissertação (Mestrado) - Escola de Engenharia da Universidade Federal de Minas Gerais (UFMG), 2006.

CLEMENT, T.P RT3D, A modular computer code for simulating reactive multi-species transport in 3dimensional groundwater systems. version 1.0, Pacific Northwest National Laboratory, U.S. Department of Energy, PNNL-11720, 1997. 59 p.
EIA/RIMA - Estudo de impacto ambiental e relatório de impacto ambiental do aterro sanitário de Uberlândia, 1996.

GODINHO-CASTRO, A.P.; TESTOLIN, R.C.; JANKE, L.; CORREAA, A.X.R.; RADETSKI, C.M. Incorporation of gypsum waste in ceramic block production: proposal for a minimal battery of tests to evaluate technical and environmental viability of this recycling process. Waste Management, v. 32, n. 1 , p. $153-157,2012$.

HARBAUGH, A.W. MODFLOW-2005, U.S. Geological survey modular ground-water model-the ground-water flow process: U S Geological Techniques and Methods 6A16, 2005, $253 \mathrm{p}$.

JACOMINO, V.F., TADDEI, M.H.T.; COTA, S.D.S.; SILVA, D.F.; MELLO, J.W.V. Avaliação do risco de contaminação ambiental decorrente do uso de fosfogesso em aterros sanitários. In: CONGRESSO DE GEOLOGIA DE ENGENHARIA E AMBIENTAL, 13; 2011, São Paulo. Anais... São Paulo: Associação Brasileira de Geologia de Engenharia e Ambiental, 2011. 1 CD.

MÁDUAR, M. F., CAMPOS, M.P., MAZZILLI, B. P., VILLAVERDE, F. L. Assessment of external gamma exposure and radon levels in a dwelling constructed with phosphogypsum plates. Journal of Hazardous Materials, v. 190, p. 1063-1067, 2011. 
OLIVEIRA, K.A.P. Aplicação do fosfogesso na agricultura do cerrado e suas implicações radiológicas. Belo Horizonte, MG, 2008. 128 f. Dissertação de Mestrado. Centro de Desenvolvimento da Tecnologia Nuclear.

PAPASTEFANOU, C.; STOULOS, S.; IOANNIDOU, A.; MANOLOPOULOU, M. The application of phosphogypsum in agriculture and the radiological impact. Journal of Environmental Radioactivity, v.89, p.188-198, 2006.

PÉREZ - LÓPES, R.; ÁLAVAREZ-VALERO, A.M.; NIETO, J.M. Changes in mobility of toxic elements during the production of phosphoric acid in the fertilizer industry of Huelva (SW Spain) and environmental impact of phosphogypsum wastes. Journal of Hazardous Materials, v.148, n. 3, p. 745-750, 2007.

PONTEDEIRO, E.M.B.D. Avaliação de modelos de impacto ambiental para deposição de resíduos sólidos contendo radionuclídeos naturais em instalações mínero-industriais. Rio de Janeiro, RJ, 2006. 167p. Tese de Doutorado. Universidade Federal do Rio de Janeiro.

SANTOS, A.J.G. Avaliação do impacto radiológico ambiental do fosfogesso brasileiro e lixiviação de Ra-226 e Pb210. São Paulo, SP, 2002. 166 f. Tese de Doutorado. Instituto de Pesquisas Energéticas e Nucleares, Universidade de São Paulo.

SHEPPARD, M. I. \& THIBAULT, D. H. Default soil solid liquid partition-coefficients, Kds, for 4 major soil types - a compendium. Health Physics, v. 59, n. 4, p. 471- 482, 1990.
SHIEH, C.S. Application of phosphogypsum in landfills. Disponível

http://www.fipr.state.fl.us/5b_Shieh_testimony.pdf. Acesso em 25/10/2011.

THORNTHWAITE, C. W., MATHER, J.R. The water balance. Centerton, NJ: Drexel Institute of Technology - Laboratory of Climatology, 1955. 104p. Publications in Climatology, vol. VIII, n.1.

US ENVIRONMENTAL PROTECTION AGENCY. Partition coefficients for metals in surface water, soil and waste. United States Environmental Protection Agency, Office of Research and Development, 2005. EPA/600/R-05/074.

VOGEL, T., HUANG K., VAN GENUTCHTEN, M.T.H. The HYDRUS code for simulating one-dimensional water flow, solute transport, and heat movement in variably-saturated media, version 5.0. Research Report No. 140, U.S. Salinity Laboratory, U.S. Department of Agriculture, 1996

WHO - WORLD HEALTH ORGANIZATION. Guidelines for drinking water quality. Third edition incorporating the first and second addenda. Volume 1, Recommendations, Geneva, 668 p. 2008.

YU, C.; LOUREIRO, C.; CHENG, J.-J.; JONES, L. G.; WANG, Y. Y.; CHIA, Y. P.; FAILLACE, E. Data collection handbook to support modeling impacts of radioactive material in soil. Illinois, USA, Argonne National Laboratory, $158 \mathrm{p}, 1993$. 